\title{
Les étrangers d'Afrique de l'Ouest à Lomé (Togo) : identification, visibilité et citadinité
}

Réflexions au regard de la ville d'Accra (Ghana)

\section{Amandine Spire}

\section{OpenEdition}

\section{Journals}

Édition électronique

URL : http://journals.openedition.org/cdg/2319

DOI : $10.4000 /$ cdg. 2319

ISSN : 2107-7266

\section{Éditeur}

UMR 245 - CESSMA

\section{Référence électronique}

Amandine Spire, "Les étrangers d'Afrique de l'Ouest à Lomé (Togo) : identification, visibilité et citadinité », Carnets de géographes [En ligne], 1 | 2010, mis en ligne le 01 octobre 2010, consulté le 24 septembre 2020. URL : http://journals.openedition.org/cdg/2319; DOI : https://doi.org/10.4000/cdg 2319

Ce document a été généré automatiquement le 24 septembre 2020.

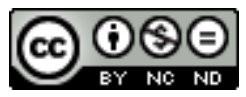

La revue Carnets de géographes est mise à disposition selon les termes de la Licence Creative Commons Attribution - Pas d'Utilisation Commerciale - Pas de Modification 4.0 International. 


\section{Les étrangers d'Afrique de l'Ouest à Lomé (Togo) : identification, visibilité et citadinité}

Réflexions au regard de la ville d'Accra (Ghana)

\section{Amandine Spire}

Cette thèse s'interroge sur les interactions entre villes et étrangers, à la lumière du contexte ouest-africain. Elle débute par un constat: les étrangers originaires de l'espace de «libre circulation» de la CEDEAO (Communauté Économique des États d'Afrique de l'Ouest) sont nombreux à Lomé et Accra et pourtant peu visibles, en raison notamment de l'absence de quartier ethnique. Malgré leur faible visibilité, certains groupes étrangers originaires de l'espace ouest-africain sont paradoxalement stigmatisés par les populations hôtes en temps de crise. 


\section{Lomé, Accra et l'espace de libre circulation de la CEDEAO (Communauté Économique des États d'Afrique de l'Ouest)}

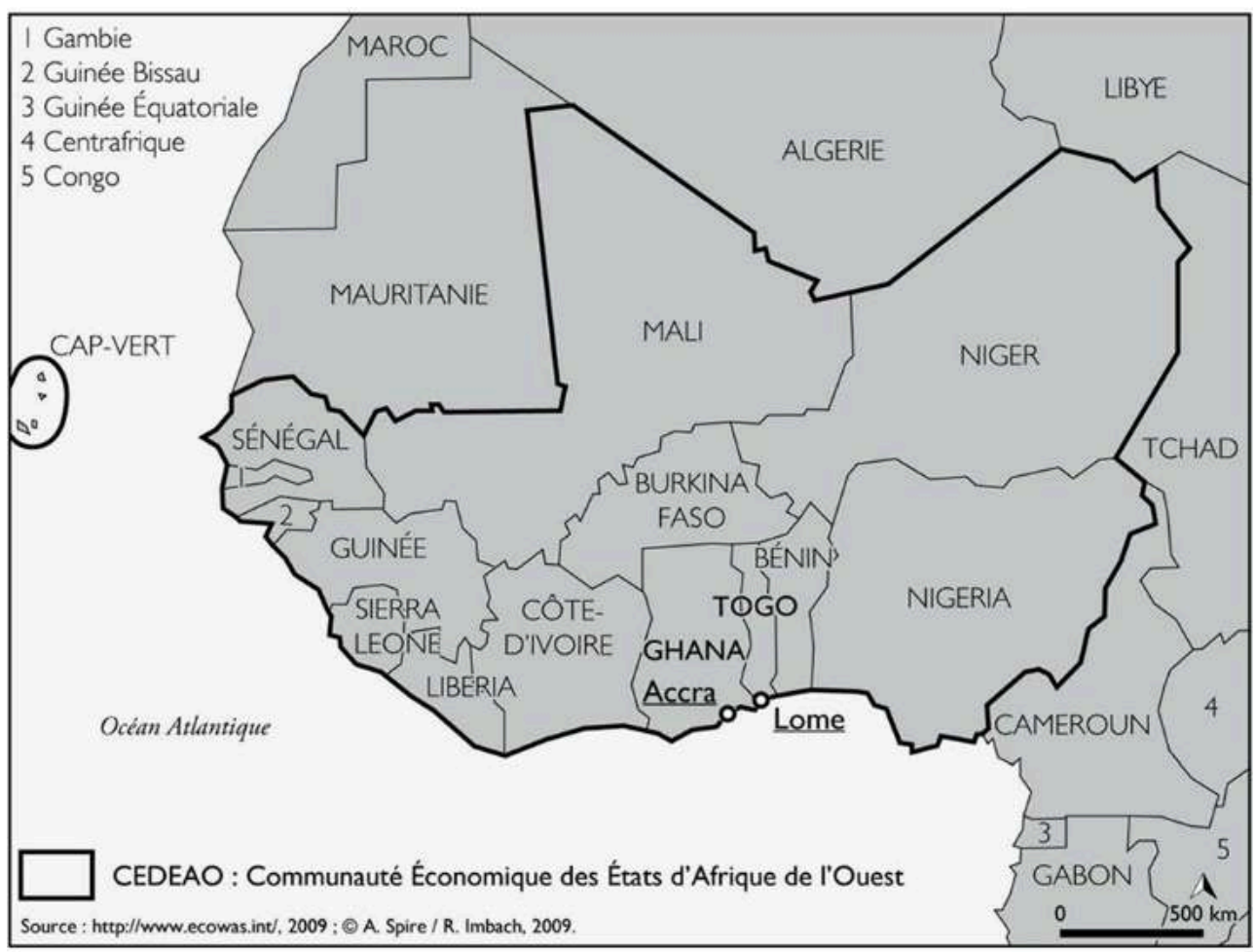

2 L'étude des sociétés urbaines de Lomé et Accra m'a conduit à remettre en cause le paradigme de l'assimilation des migrants à la ville tel que conçu dans la tradition sociologique de Chicago. En m'appuyant sur les théories de l'École de Manchester et en déconstruisant le modèle de l'exode rural, j'ai proposé une approche dialectique et multiscalaire des liens entre étrangers et villes : la ville modifie les identifications des migrants étrangers qui, eux-mêmes, transforment les espaces de la ville et la citadinité, définie comme les manières d'être propres à une ville (en termes de pratiques et de représentations).

3 Un des apports principaux de ma thèse consiste à souligner la complexité de la notion d'étranger dans les villes d'Afrique de l'Ouest. La diversité des mobilités internationales, par les lieux et les temporalités convoqués, participe à brouiller les visages des étrangers à Lomé et à Accra. Les étrangers n'appartiennent pas à un seul groupe social, pas plus qu'à un seul territoire. Aussi, cette recherche s'interroge-t-elle sur la visibilité des identifications étrangères à la ville, tant aux yeux des citadins qu'à ceux du chercheur.

4 Les identités étrangères à une ville peuvent être l'objet d'une revendication et d'un processus de reconnaissance qui se traduit par des territorialités à l'échelle d'un quartier comme c'est le cas dans les quartiers zongo. Mais la marginalité de certains groupes étrangers contribue également à la formation de territorialités étrangères, dans le registre invisible. La présence des étrangers s'exprime non seulement en termes identitaires, mais aussi territoriaux. La prise de possession et le contrôle de certains espaces par les étrangers sont au cœur de dynamiques syncrétiques caractérisées par la 
redéfinition d'appartenances à l'ailleurs dans des interactions locales. Autrement dit, le maintien d'identités étrangères à la ville ne repose pas sur la réplique d'identités qui apparaissent ailleurs ou dans d'autres temps mais semblent bien le produit d'une différenciation et d'une création identitaire dans et de la ville. Il est dès lors possible de dépasser la dimension territoriale des changements de la ville liés à la présence des étrangers : à l'échelle micro, des lieux de sociabilités créés par les étrangers participent pleinement à inventer les liens qui font la ville au quotidien.

\section{Méthode de recherche}

La thèse s'appuie sur un matériau empirique collecté au cours d'un terrain d'une durée d'un an, réalisé entre 2007 et 2008. La pratique du terrain mobilise une méthode proche de l'ethnographie, articulant observation et entretiens menés, autant que possible, en anglais ou en français. Dans les deux villes, deux niveaux d'observation ont schématiquement été distingués. D'une part, les espaces publics composés principalement de la rue, des marchés et des gares routières ont été l'objet d'une analyse propre. D'autre part, Les espaces privés ou semi-privés formés par les espaces domestiques (internes et externes) et certains lieux commerciaux, ont été étudiés grâce à une familiarisation grandissante avec le terrain. Les enquêtes ont été réalisées conjointement avec trois grandes catégories d'interlocuteurs : les représentants de la ville (chefs de quartiers, chefs de groupes étrangers, leaders d'association de ressortissants), des citadins se considérant comme étrangers et enfin des citadins non étrangers (afin de saisir la perception et les représentations des migrants). La recherche repose sur la confrontation de terrains considérés aussi pertinents que tout autre pour apporter une contribution à la réflexion sur les liens entre migrants étrangers et dynamiques urbaines en Afrique de l'Ouest. Le choix de mettre en perspective les cas de Lomé et Accra n'a pas pour objectif de démontrer l'analogie entre deux objets d'étude, mais d'enrichir l'étude de l'une des villes par une confrontation avec un second terrain afin de tenter de monter en généralité. La confrontation permet d'accentuer les spécificités ou les processus en œuvre au cas par cas, tout en relativisant les facteurs déterminants.

\section{Fiche informative}

Discipline

Géographie

\section{Directeur}

M. Philippe Gervais-Lambony

\section{Université}

Université Paris-Ouest Nanterre, laboratoire GECKO.

Membres du jury de thèse, soutenue le 16 novembre 2009

- Michel Agier, rapporteur, EHESS

- Alain Dubresson, Université Paris Ouest Nanterre

- Philippe Gervais-Lambony, directeur, Université Paris Ouest Nanterre

- Émile Le Bris, directeur de recherches à l'IRD 
- Emmanuel Ma Mung, rapporteur, directeur de recherches CNRS

- Gabriel Nyassogbo, Université de Lomé (Togo)

Situation professionnelle actuelle

ATER à l'Université de Paris-Sorbonne

Contact de l'auteur

Amandinespire[at]gmail.com

INDEX

Thèmes : Carnets de soutenances 